\title{
Franco Selleri and the rotating disk
}

\author{
Alexander Afriat
}

August 26, 2018

\begin{abstract}
I concentrate on the pars destruens, rather than the pars construens, of Selleri's work on the Sagnac effect. He speaks (2003) of the "impossibilità di spiegare la fisica sulla piattaforma ruotante con la TRS," and may have a point. By confining our attention to the world-cylinder above a circle on the disk we avoid broader integrability issues that just cause confusion. A rate of rotation foliates the cylinder into timelike spirals, and also into the simultaneity spirals hyperbolically orthogonal to them; together the two foliations give rise to all sorts of temporal absurdities.
\end{abstract}

\section{Introduction}

Selleri's work on the Sagnac effect suggests that rotation is enough to produce relativistic temporal absurdities - the effect can be accounted for in the laboratory frame, but not with respect to the very disk on which it is produced.

Simultaneity in relativity is relative to the velocity of the observer; different observers foliate space-time in different ways. Simultaneity can be understood globally, 'rigidly': a timelike four-vector $V_{0}$ at, say, the origin of flat space-time $M$ can be taken to foliate all of $M$ into flat simultaneity surfaces hyperbolically orthogonal to $V_{0}$. Or it can be understood locally: a timelike vector $V_{x} \in T_{x} M$ at $x \in M$ foliates the tangent space $T_{x} M$ into simultaneity subspaces of a local character. The metric

$$
\eta^{b}: T_{x} M \rightarrow T_{x}^{*} M
$$

turns a vector $V_{x}$ into the covector $V_{x}^{b}=\eta^{b} V_{x}$ whose level surfaces themselves foliate $T_{x} M$ into simultaneity spaces; a timelike vector field $V$ on $M$ is thus transformed into a one-form $V^{b}$ on $M$ representing a distribution of simultaneity spaces. But do the local spaces fit together so as to make global sense, yielding a broadly valid notion of time? Are they integrable? Can they give rise to anholonomies, absurdities, departures from integrability that prevent a satisfactory account of the Sagnac effect as seen from the disk?

\section{Cylinder}

A rotating disk is bound to complicate or obstruct integration. But many integrability issues just cause confusion; to simplify, we can confine our attention to a single circle 
$C_{r}$-say of radius $r=1$ - of the disk, and to the world-cylinder $\mathbf{C}=\mathbf{C}_{1} \subset M$ it describes. Each tangent space $T_{x} \mathbf{C}$ is a two-dimensional subspace of $T_{x} M$; the vector $V_{x}$ can be replaced by the projection $\mathbf{V}_{x} \in T_{x} \mathbf{C}$ characterised by a (length and) polar angle $\varphi$, which vanishes for the laboratory. The corresponding simultaneity line $\mathbf{L}_{x}(\bar{\varphi}) \subset T_{x} \mathbf{C}$ is a ray with polar angle

$$
\bar{\varphi} \equiv \frac{\pi}{2}-\varphi
$$

where the bar expresses the hyperbolic orthogonality ( ' $\varphi \perp \bar{\varphi}$ ') due to the signature of $\eta$, and light travels at $\pm \pi / 4$. The projection $\mathbf{V}_{x}^{b} \in T_{x}^{*} \mathbf{C}$ of the covector $V_{x}^{b}$ foliates $T_{x} \mathbf{C}$ into lines parallel to $\mathbf{L}_{x}(\bar{\varphi})$. So a given rate of rotation induces a double foliation of $\mathbf{C}$ : into the spiral lines

$$
\mathbf{A}(\varphi)=\left\{\mathbf{A}^{m}(\varphi)\right\}_{m}
$$

along the vector field $\mathbf{V}$; and into the corresponding simultaneity lines

$$
\mathbf{S}(\bar{\varphi})=\left\{\mathbf{S}^{n}(\bar{\varphi})\right\}_{n},
$$

all of which are everywhere orthogonal to the spirals of $\mathbf{A}(\varphi)$. The real numbers $m$ and $n$ somehow parametrise the spirals of their respective foliations. We can use Cartesian coordinates $(x, y, z, t)_{\mathscr{L}}$ in the laboratory frame, confine the disk to the plane where $z$ vanishes, and stipulate that the distinguished spirals $\mathbf{A}^{0}(\varphi)$ and $\mathbf{S}^{0}(\bar{\varphi})$ pass through $(1,0,0,0)_{\mathscr{L}}$; the other values of $m$ and $n$ can then be times in the lab frame. Alternatively we can use coordinates $(\alpha, r, z, t) \mathscr{L}^{\prime}$, where the azimuth $\alpha$ vanishes at $(1,0, z, t)_{\mathscr{L}}$. If $\alpha$ is used to parametrise the spirals of the two foliations we can use the same symbols $\mathbf{A}^{0}(\varphi)$ and $\mathbf{S}^{0}(\bar{\varphi})$ for the same distinguished spirals, which both pass through the event

$$
(0,1,0,0)_{\mathscr{L}^{\prime}} \leftrightarrow(1,0,0,0)_{\mathscr{L}}
$$

But time on its own isn't enough to parametrise everything, nor is the azimuth. The laboratory's simultaneity circles

$$
\mathbf{S}(\overline{0})=\left\{\mathbf{S}^{t}(\overline{0})\right\}_{t}
$$

can be parametrised by the lab time $t$, whereas the azimuth is best for a parametrisation of the vertical worldlines

$$
\mathbf{A}(0)=\left\{\mathbf{A}^{\alpha}(0)\right\}_{\alpha} .
$$

We're already in trouble: the rotating observer following $\mathbf{A}^{0}(\varphi)$ cuts the simultaneity spiral $\mathbf{S}^{0}(\bar{\varphi})$ at $(1,0,0,0)_{\mathscr{L}}$, and periodically thereafter (and indeed before); the intersections are both simultaneous and successive; but how can they be?

\section{Sagnac}

Einstein (1916) considers an optical pulse $\Pi$ emitted from the middle $\sigma$ of a train carriage. Even if, seen from the station, $\Pi$ reaches the trailing end first, it reaches both ends at the same time with respect to the motion of the train-and if the rays are reflected back from the ends, they return to the source $\sigma$ at the same time. 
Sagnac's experiment amounts to bringing the ends of Einstein's carriage together by bending it into a circle, say $C_{1}$ : light rays from a common source are sent around a rotating disk in opposite directions; an interference pattern-which can be taken to measure rotation-indicates the phase difference when the rays return to the source. Selleri $(2001,2003,2004,2012)$ contended that standard relativity theory, with its relativity of simultaneity, cannot account for the effect with respect to the very disk on which it is produced.

Einstein's neatly intersecting straight worldlines get bent in our cylindrical environment, where the rays rise along the various local light cones (or rather $\pm \pi / 4$ 'crosses' $\in T_{x} \mathbf{C}$ ) and intersect periodically, 'every half-lap'; all of which is invariant and has nothing to do with the rotation of the disk. Without rotation, the second optical intersection $\mu$ (after a full lap) falls on the source's line $\mathbf{A}^{0}(0)$, where the azimuth vanishes. Rotation splits the single intersection $\mu$ into two separate intersections, $\mu_{c} \&$ $\mu_{a}$ : anticlockwise rotation, for instance, makes the source's spiral $\mathbf{A}^{0}(\varphi)$ intersect the clockwise ray, at

$$
\mu_{c} \in \mathbf{S}^{t_{c}}(\overline{0}),
$$

before the anticlockwise ray, at

$$
\mu_{a} \in \mathbf{S}^{t_{a}}(\overline{0}) .
$$

\section{Lapse}

The intersections $\mu_{c} \& \mu_{a}$ are simply events, invariant spatiotemporal events, which are independent of any particular foliation or representation of time. But once we want to evaluate the time elapsed between them, we need to embed them in an appropriate temporal structure, to extract pure time from space\&time. The laboratory provides one foliation, which gives one answer

$$
\Delta t=t_{a}-t_{c}
$$

for the lapse, where $t_{a}$ and $t_{c}$ are the time values of the lab circles $\mathbf{S}^{t_{a}}(\overline{0})$ and $\mathbf{S}^{t_{c}}(\overline{0})$. But even Einstein's pulse reached the trailing end first with respect to the train station; so far, rotation offers nothing new.

Suppose we confine our attention to the two intersections $\mu_{c} \& \mu_{a}$, ignoring the rest of the cylinder and any global issues that may arise. The spiral segment $\gamma$ joining them, of length $\lambda$, is short enough to be well approximated by the tangent vector $\dot{\gamma} \in T_{\mu_{c}} \mathbf{C}$ of the same length

$$
\lambda=\sqrt{\eta(\dot{\gamma}, \dot{\gamma})} .
$$

The time lapse

$$
\Delta \tau=\left\langle\mathbf{V}^{b}, \dot{\gamma}\right\rangle
$$

will be given by the covector $\mathbf{V}^{b} \in T_{\mu_{c}}^{*} \mathbf{C}$ representing the local temporal structure corresponding to a certain rate of rotation. This lapse will in fact have a double dependence on the rate: it is after all rotation that splits $\mu$ and pushes apart $\mu_{c} \& \mu_{a}$ in the first place; and the time lapse $\Delta \tau$ between two intersections can then be calculated 
with respect to the very rate $\varphi$ that produced the separation, or even to any other rate $\varphi^{\prime}$. To be explicit one could write

$$
\Delta \tau\left(\varphi, \varphi^{\prime}\right)=\left\langle\mathbf{V}^{b}\left(\varphi^{\prime}\right), \dot{\gamma}(\varphi)\right\rangle
$$

Even if the disk is spinning so fast that the spiral segment $\gamma$ between $\mu_{c} \& \mu_{a}$ is too curved to be well approximated by the tangent vector $\dot{\gamma}$, the intersections are still contained in a simply connected region on which the temporal one-form $\mathbf{V}^{b}$ is closed; so that whatever integration may be needed to evaluate the lapse $\Delta \tau$ won't cause trouble (by producing anholonomies).

But there's more to the cylinder than just the intersections $\mu_{c} \& \mu_{a}$. Even if one insists on viewing the evaluation of $\Delta \tau(\varphi, \varphi)$ as a largely local matter, the rest of the cylinder is still there, with its troublesome orthogonal foliations $\mathbf{A}(\varphi)$ and $\mathbf{S}(\bar{\varphi})$ which can be used to generate all sorts of absurdities: Every spiral $\mathbf{S}^{n}(\bar{\varphi})$ represents a particular instant; every turn of the spiral, for instance every intersection of $\mathbf{S}^{n}(\bar{\varphi})$ with $\mathbf{A}^{\alpha}(0)$, therefore represents the same instant. So $\mu_{a}$, or any other event, happens before it happens-being below all the subsequent turns in the same spiral-and also after it happens-being above all the previous turns in the same spiral. By manipulating and combining such temporal absurdities one can give the intersections $\mu_{c} \& \mu_{a}$ a time lapse, with respect to $\varphi^{\prime}$ (for instance $\varphi^{\prime}=\varphi$ ), of both signs and every size. Ex absurdo quodlibet.

The right not to look beyond $\mu_{c} \& \mu_{a}$ seems questionable; and the opposite rightto look beyond the two intersections-is hard to deny if insisted upon. So at best there is a choice: to ignore almost all of the cylinder, or not.

\section{Final remarks}

To avoid the problems Selleri had in mind, almost all reference frames would have to be ruled out: rotating frames? all accelerated, curvilinear frames? Leaving only coordinate systems that diagonalise (with \pm 1 ) the Minkowski metric $\eta$ ?

Special relativity has always been a theory of flat space-time; in the early years severe restrictions on reference frames were sometimes added, even by Einstein himself. But it has since emerged, certainly since Kretschmann (1917), as a theory whose flat space-time can be described by a large class of generally curvilinear coordinate systems. Selleri's work on the Sagnac effect obliges us, it would seem, to impose the most awkward restrictions on the class of reference frames-if the relativity of simultaneity is not to be given up altogether.

Three stances come to mind:

1. We just evaluate the lapse $\Delta \tau$ locally (with respect to some rate $\varphi^{\prime}$ or other), confining our attention to $\mu_{c} \& \mu_{a}$ (a single tangent space, or little more)ignoring the rest of the cylinder, and all the temporal absurdities it involves.

2. We rule out almost all reference frames, keeping only a handful.

3. We exhume the æther Einstein got rid of, assigning it the only foliation allowed and giving up the relativity of simultaneity. 
I thank the Centro di Ricerca Matematica Ennio De Giorgi, Scuola Normale Superiore, for generous hospitality and support; and Ermenegildo Caccese and Antonio Masiello for many conversations about rotation in relativity.

\section{References}

Einstein, A. (1916) Über die Spezielle und die Allgemeine Relativitätstheorie, Vieweg, Berlin

Kretschmann, E. (1917) "Über den physikalischen Sinn der Relativitätspostulate, A. Einsteins neue und seine ursprüngliche Relativitätstheorie" Annalen der Physik 53, 576-614

Selleri, F. (2001) "Relatività e relativismo" Revista de Filosofia 25, 23-51

Selleri, F. (2003) "Sagnac effect: end of the mystery" in Relativity in rotating frames, edited by G. Rizzi and M. Ruggiero, Kluwer

Selleri, F. (2004) "Relativismo ed etere di Lorentz" pp. 16-36 in Atti del XXIII Congresso nazionale di Storia della fisica e dell'astronomia, edited by P. Tucci, A. Garuccio and M. Nigro, Progedit, Bari

Selleri, F. (2012) "The Sagnac effect, once more" pp. 525-7 in Proceedings of the NPA, Albuquerque, NM 